\title{
Research on the Novel Phosphorus Flame Retardant Epoxy Resin Model and the Corresponding Flame Retardant Performance
}

\author{
Jitang Zhang, Jicai Liang, Wanxi Zhang*
}

College of materials science and engineering, Jilin university, Changchun, Jilin, 130025, China.

\begin{abstract}
Keywords: Flame Retardant, Performance, Retardant Epoxy, Resin Model, Novel Structure.
\end{abstract}
\begin{abstract}
This paper conducts the research on the novel phosphorus flame retardant epoxy resin model and that the corresponding flame retardant performance. Research will be introduced to the phosphorous epoxy resin curing agent of the structure, synthesis of phosphorus curing agent, curing agent with flame retardant performance. The way of preparation of phosphorus curing agents of effect is very good, but relatively cumbersome process for synthesizing problems and the cured epoxy resin although the flame retardant performance is good, but the relative matrix resin, its glass transition temperature and mechanical properties are decreased. Under this concern, this paper integrates the literature review to propose the novel phosphorus flame retardant epoxy resin model and that the corresponding flame retardant performance. We conduct experiment to verify general performance and effectiveness of the material that is meaningful.
\end{abstract}

\section{Introduction}

Epoxy resin is kind of thermosetting polymer composite material with good performance that widely used in metal and non-metallic materials, corrosion resistant coatings as electrical insulation materials and in the production of the glass fiber reinforced plastic composite material. For the environmental protection requirements, halogen free flame retardant alternative technology demand is very urgent. Research shows that introducing a small amount of phosphorus in the polymer chain, not only can make the material has the flame retardant performance, and at the same time to keep a good flame retardant durability. In the figure one, we show the overview [1-2].

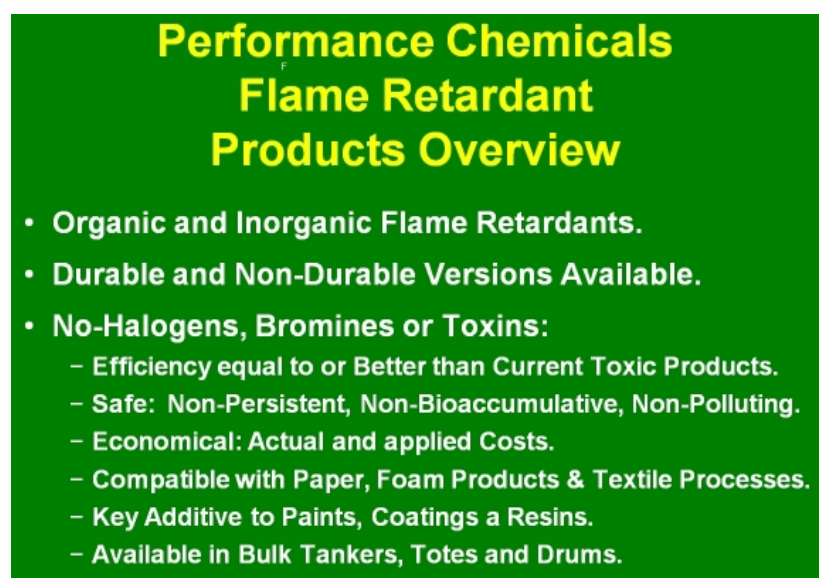

Fig. 1 The Flame Retardant Overview

At present, the most common method, improve the effect of flame retardant epoxy resin is will be introduced to the containing halogen flame retardant epoxy resin, the method is simple operation, obvious effects. According to the literature review, we summarize the existing flame retardant relate techniques as follows. (1) Organic phosphorus flame retardants. Organic phosphorus flame retardants are with phosphorus-containing flame retardants and organic fire retardant agent, many varieties, wide application including phosphate, phosphonic acid ester, phosphite ester and organic phosphorus salt, phosphine oxide, such as phosphorus polyols and phosphorus and nitrogen compounds. (2) The chlorinated flame retardants. Phosphorus-containing flame retardants for its excellent flame retardant performance, low price sales have firmly flame retardants. However phosphorus-containing flame 
retardants burn freed up a lot of black smoke and poisonous gas makes it the usage of the declining trend year by year. (3) Bromine flame retardants. Bromine flame retardants flame retardant ABS resin research started the earliest as has now developed a variety of excellent flame retardant performance. Flame retardant of more and more researchers set out to research and development or non-toxic, good thermal stability and lower toxicity and smoke less new type combustion agent. (4) Organic nitrogen flame retardant. In view of the environment requirements for flame retardant materials, high, triazine as maternal nitrogen flame retardant more and more get favor of people. Flame retardant mechanism of melamine is more complex, it is not very clear, it is considered in the heat elimination reaction step by step, to absorb a large amount of heat, and release ammonia, so as to achieve flame retardant effect. (5) Other inorganic flame retardants. Magnesium hydroxide and general aluminum hydroxide are two common packing type inorganic flame retardants its characteristic is halogen-free, non-toxic, smoke suppression and low price. Inorganic flame retardant ABS resin is to explore development direction of flame retardant effect and smoke suppression. New type of inorganic flame retardant ammonium sulfate in the ABS resin showed excellent flame retardant and smoke suppression function, when use with halogen, antimony flame retardant, has good flame retarding synergistic action.

In this manuscript, to deal with mentioned challenges, we conduct research on novel phosphorus flame retardant epoxy resin model and the corresponding flame retardant performance.

\section{Our Proposed Methodology}

Review of the Flame Retardant Resin Features. Epoxy resin in coatings, civil, building, adhesives, electronic, aviation, etc. that many fields obtained the widespread application as has become a very important a thermosetting resin. But the epoxy flammability and that away from the fire of the fat continues to make them is limited the application of the spontaneous combustion, so the preparation of flame retardant epoxy resin is one of the research topic people are interested in. So, low toxicity, low smoke zero halogen, environmental protection has become the development trend of that flame retardant products in the world. Of phosphorus flame retardant resin in the process of heating or the burning only commonly produce phosphoric acid, phosphate and water vapor, etc., its toxic and that corrosive smoke all below contain halogen flame retardant resin [3-4].

New phosphorus flame retardant epoxy resin are introduced in the structure of the epoxy resin containing phosphorus, it belongs to the nature of the flame retardant epoxy resin, don't need to be modified or flame retardant treatment that also has high temperature resistance, oxidation resistance, non-flammable, etc. different from phosphorus group flame retardant modification of epoxy resin.

Phosphorus Flame Retardant Epoxy Resin Basis. Phosphorus preparation of epoxy resin was first introduced through specific reaction in that phosphorus compound molecular double amino, single/double hydroxyl, single/double carboxyl, single/double epoxy group, such as reactive group, as to introduce phosphorus flame retardant epoxy groups the starting material, make them participate in the preparation of epoxy resin reaction, the phosphorus group according to the target type or curing epoxy resin molecular chain network structure. Sometimes to the initial molecular compounds were introduced in aromatic structure to improve the curing of the basic resin glass transition temperature, thermal stability and mechanical properties, etc.

Phosphorus groups phosphorous epoxy compound molecular structure and the general location of corresponding greater effects on the thermal properties of curing system. Different way to get the essence of phosphorus flame retardant epoxy curing system of the thermal performance of obvious differences, there are differences with general epoxy curing system. General epoxy resin that curing system of thermal degradation for the step. Phosphorus group thermal stability is poor, in early degradation under low temperature curing system is degraded, but has a promoting effect on the formation of a rich phosphorus coke residue. The residual thermal stability is better in addition to the protection of curing system as inhibit thermal oxidation reaction in base material to outside, still can improve the thermal degradation of curing system of temperature, the temperature of mass loss and coke production. Nature of phosphorous flame retardant resin systems usually cannot meet the 
requirements of several essential performances at the same time, such as flame retardant properties, heat resistance, acid-proof alkaline, high temperature does not change color, etc. Second, its thermal stability and glass transition temperature is often a certain negative impact, we need to add special components to weaken the influence and further improve the performance.

The Proposed Methodology. Type phosphorus flame retardant epoxy resin system is one of the phosphorus flame retardant systems as flame retardant mechanism including gas phase mechanism and flame retardant mechanism of condensed phase. Gas phase mechanism two aspects of physical and chemical action, physical aspects mainly refers to flame retardant decompose at high temperature to some difficult flammable, reduce the concentration of combustible gas, or due to the difficult flammable than major that will burn body in isolation effect.

Through some active monomers containing phosphorus group on modification of that the epoxy compound way to introduce the phosphorus pre polymers, epoxy resin and curing agent curing epoxy resin get phosphorous flame retardant epoxy resin, can be largely overcome the above problems, and meet the strict requirements of practical application. Although there is also a complicated synthesis technology, the cost is higher it has aroused the concern of the electronics industry, become one of the very promising way of flame retardant epoxy resin. Phosphorous epoxy resin is generalized by the method of reactive phosphorous compounds with epoxy resin or reaction is phosphorus epoxide group and form, the resin to normal epoxy resin curing agent or phosphorus curing agents of reaction can be phosphorus flame retardant epoxy resin system [5].

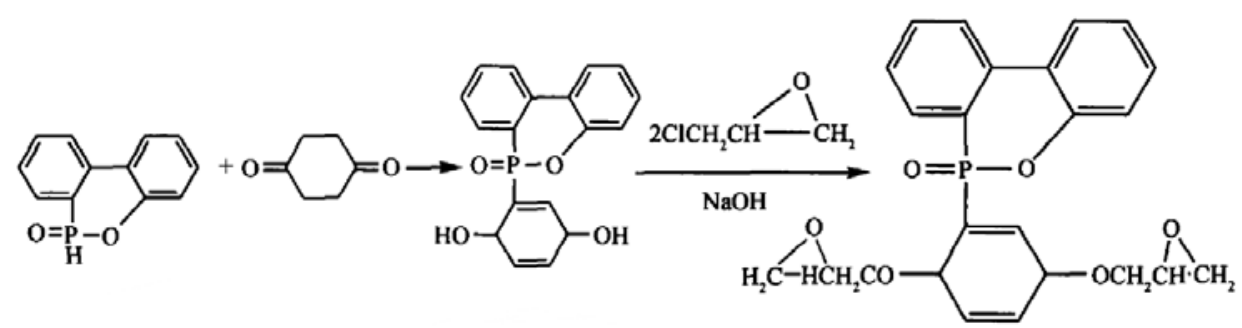

Fig. 2 The Processing of Creating the Proposed Material

Phosphorus is half cured epoxy resin containing phosphorus compounds with epoxy resin to partly formed by the ring opening reaction of epoxy groups, the half cured system can again with ordinary curing agent for epoxy resin curing and phosphorus flame retardant system. Generally speaking, after the reaction, curing, half cured formation of flame retardant system of the thermal stability, flame retardancy are better than the phosphorus directly an epoxy resin system. In the figure two, we show the classic steps of creating the corresponding material. At room temperature can be stable storage, low toxicity, simple synthesis, application of low cost, epoxy curing high electrical performance, at the same time because of the high phosphorus content rather than the other add flame retardant has excellent thermal stability and flame retardancy that epoxy base material is very potential.

\section{Performance Evaluation Test}

To verify the performance of the proposed method, we conduct experimental analysis in this part, the parameter one represents the resin decomposition temperature of loss when the mass fraction of 5\% and the parameter two denotes the thermal maximum weight loss rate of the temperature. 
Table. 1 The Experimental Result of the Proposed Methodology

\begin{tabular}{|c|c|c|}
\hline Experimental Set & Parameter One & Parameter Two \\
\hline 1 & 371 & 395 \\
\hline 2 & 377 & 399 \\
\hline 3 & 375 & 398 \\
\hline 4 & 374 & 396 \\
\hline 5 & 370 & 392 \\
\hline 6 & 375 & 390 \\
\hline 7 & 372 & 397 \\
\hline 8 & 376 & 392 \\
\hline 9 & 371 & 395 \\
\hline 10 & 371 & 398 \\
\hline 11 & 373 & 401 \\
\hline 12 & 372 & 395 \\
\hline 13 & 373 & 396 \\
\hline 14 & 370 & 394 \\
\hline 15 & 376 & 399 \\
\hline
\end{tabular}

\section{Summary and Conclusion}

In this paper, we conduct research on the novel phosphorus flame retardant epoxy resin model and the corresponding flame retardant performance. In recent years, based on the need of the environmental protection, safety, environment friendly flame retardant epoxy resin has become the general research development and application of the hot spots, especially the reactive flame retardant epoxy resin. As one of the three largest general-purpose thermosetting resin, the epoxy resin because of its high light transmittance, good mechanical performance, large refractive index, corrosion resistance, excellent electric properties and the low cost is widely applied in various fields. Under this basis, this paper proposes the novel perspectives on the issues that are innovative.

\section{Acknowledgement}

This paper is supported by the development of science and technology plan projects of department of science in Jilin (20150301002GX).

\section{References}

[1] Gu, Hongbo, et al. "Flame-retardant epoxy resin nanocomposites reinforced with polyaniline-stabilized silica nanoparticles." Industrial \& Engineering Chemistry Research 52.23 (2013): 7718-7728.

[2] Zhang, Wenchao, et al. "The influence of the phosphorus-based flame retardant on the flame retardancy of the epoxy resins." Polymer Degradation and Stability 109 (2014): 209-217.

[3] Dogan, Mehmet, and S. Murat Unlu. "Flame retardant effect of boron compounds on red phosphorus containing epoxy resins." Polymer Degradation and Stability 99 (2014): 12-17.

[4] Wang, Qiuping, et al. "Synthesis of a novel polysiloxane containing phosphorus, and boron and its effect on flame retardancy, mechanical, and thermal properties of epoxy resin." Polymer Composites (2016).

[5] Yu, Bin, et al. "Enhanced thermal and flame retardant properties of flame-retardant-wrapped graphene/epoxy resin nanocomposites." Journal of Materials Chemistry A 3.15 (2015): 8034-8044. 